\title{
Ultrathin-shell epitaxial Ag@Au core-shell nanowires for high-performance and chemically-stable electronic, optical, and mechanical devices
}

\author{
Yangzhi Zhu ${ }^{1,2}$, Sanggon Kim ${ }^{1}$, Xuezhi $\mathrm{Ma}^{3}$, Peter Byrley ${ }^{1}$, Ning $\mathrm{Yu}^{1}$, Qiushi $\mathrm{Liu}^{3}$, Xiaoming Sun ${ }^{4}$, Da Xu ${ }^{3}$, \\ Sangshan Peng ${ }^{5}$, Martin C. Hartel ${ }^{6}$, Shiming Zhang ${ }^{7}$, Vadim Jucaud $^{2}$, Mehmet R. Dokmeci ${ }^{2}$, Ali Khademhosseini ${ }^{2}$, \\ and Ruoxue Yan $^{1,8}(\bowtie)$ \\ ${ }^{1}$ Department of Chemical and Environmental Engineering, Bourns College of Engineering, University of California-Riverside, Riverside, CA \\ 92521, USA \\ ${ }^{2}$ Terasaki Institute for Biomedical Innovation, Los Angeles, CA 90064, USA \\ ${ }^{3}$ Department of Electrical and Computer Engineering, University of California-Riverside, Riverside, CA 92521, USA \\ ${ }^{4}$ State Key Laboratory of Chemical Resource Engineering, Beijing University of Chemical Technology, Beijing 100029, China \\ ${ }^{5}$ State Key Laboratory of Chemo/Biosensing and Chemometrics, College of Chemistry and Chemical Engineering, Hunan University, \\ Changsha 410082, China \\ ${ }^{6}$ Department of Bioengineering, University of California-Los Angeles, Los Angeles, CA 90095, USA \\ ${ }^{7}$ Department of Electrical and Electronic Engineering, The University of Hong Kong, Hong Kong, China \\ ${ }^{8}$ Material Science and Engineering Program, University of California-Riverside, Riverside, CA 92521, USA
}

(C) The Author(s) 2021

Received: 23 April 2021 / Revised: 22 June 2021 / Accepted: 23 June 2021

\begin{abstract}
Silver nanowires (AgNWs) hold great promise for applications in wearable electronics, flexible solar cells, chemical and biological sensors, photonic/plasmonic circuits, and scanning probe microscopy (SPM) due to their unique plasmonic, mechanical, and electronic properties. However, the lifetime, reliability, and operating conditions of AgNW-based devices are significantly restricted by their poor chemical stability, limiting their commercial potentials. Therefore, it is crucial to create a reliable oxidation barrier on AgNWs that provides long-term chemical stability to various optical, electrical, and mechanical devices while maintaining their high performance. Here we report a room-temperature solution-phase approach to grow an ultra-thin, epitaxial gold coating on AgNWs to effectively shield the Ag surface from environmental oxidation. The Ag@Au core-shell nanowires (Ag@Au NWs) remain stable in air for over six months, under elevated temperature and humidity $\left(80{ }^{\circ} \mathrm{C}\right.$ and $100 \%$ humidity) for twelve weeks, in physiological buffer solutions for three weeks, and can survive overnight treatment of an oxidative solution $\left(2 \% \mathrm{H}_{2} \mathrm{O}_{2}\right)$. The Ag@Au core-shell NWs demonstrated comparable performance as pristine AgNWs in various electronic, optical, and mechanical devices, such as transparent mesh electrodes, surface-enhanced Raman spectroscopy (SERS) substrates, plasmonic waveguides, plasmonic nanofocusing probes, and high-aspect-ratio, high-resolution atomic force microscopy (AFM) probes. These Au@Ag core-shell NWs offer a universal solution towards chemically-stable AgNW-based devices without compromising material property or device performance.
\end{abstract}

\section{KEYWORDS}

epitaxial growth, core-shell nanowire, plasmonic waveguides, atomic force microscopy (AFM) probe, transparent electrode, wearable electronics

\section{Introduction}

Silver nanowires (AgNWs) are known to have the highest electrical and thermal conductivity among metal nanowires, garnering significant attention for their use in transparent conductive films, including thin-film solar cells, supercapacitors, transparent heaters, and touch panels [1-6]. In addition, penta-twinned AgNWs synthesized through solution-phase polyol methods also show a low ohmic loss, strong plasmonic enhancement, high mechanical strength, and flexibility due to their inherently high crystallinity, low impurity level, and atomically flat surfaces $[7,8]$. These characteristics make them promising candidates for emerging applications in stretchable electronics and optoelectronics, photonic-plasmonic circuits, surface-enhanced Raman spectroscopy (SERS) sensors, and various high-resolution scanning probe microscopies (SPM) [9-15].

However, a daunting obstacle for device-level commercialization of AgNWs is their vulnerability to oxidation, especially under heat, light, and moisture [16, 17], which causes severe deterioration to the mechanical, electrical, and optical properties of AgNWs and leads eventually to device failure. Therefore, there has been an ongoing drive to develop strategies for the effective oxidation protection of AgNWs without impairing their inherent electrical, optical, and mechanical properties, preferably through low-cost solution-phase processes to be 
compatible with large-scale industrial device fabrication. So far, approaches reported to address the long-term stability of AgNWs could be summarized into two major categories: (1) For devices fabricated with AgNW networks, a polymer or ceramic overlayer is coated on the AgNW networks predeposited on a substrate as a corrosion barrier, such as polydimethylsiloxane (PDMS) [18-21], poly (methyl methacrylate) (PMMA) [22, 23], poly (3,4-ethylenedioxythio-phene): poly(styrene sulfonate) (PEDOT: PSS) [24-27], reduced graphene oxide (rGO) [28-30] and atomic-layer-deposited aluminumdoped zinc oxide $(\mathrm{AZO})$ and aluminum oxide $\left(\mathrm{Al}_{2} \mathrm{O}_{3}\right)[2,31,32]$; (2) A relatively stable metal or carbon shell, such as $\mathrm{Ni}$ [33-35], C [36-38], Zn [39, 40], Sn [41, 42], Au [43-46] and Pt [47] is chemically grown on the AgNW surface through a solutionphase process before device fabrication. Both methods have shown effectiveness in prolonging the lifetimes of AgNW mesh electrodes. However, many other applications, such as surface plasmon subwavelength optics, SERS sensors, and SPM probes, have more stringent requirements on the coating material property, thickness, surface smoothness, interface quality, and surface chemistry to maintain the high device performance. For example, a rough coating or a rough interface between the shell and Ag core would result in a significant increase in surface/interface electron scattering that adds detrimental optical loss to surface plasmon waveguides [7]. Since surface plasmons are very sensitive to slight perturbations within the skin depth, the shell material needs to be as conductive as possible and the coating as thin and uniform as possible to minimize additional ohmic loss and maintain the high performance of AgNWs in subwavelength optics and SERS sensors. However, up till now, it remains a significant challenge to accurately control the coating thickness, uniformity, interface, and surface smoothness to allow reliable oxidation protection in various working environments (e.g., under light, heat, humidity, and in solution) while keeping the effects on optical, electrical, and mechanical properties of AgNWs to a minimum. Therefore, there is an urgent need to develop a universal, economic, and scalable approach to expand the success in the antioxidation treatments of AgNW mesh electrodes to a wide variety of AgNW-based devices.

To satisfy the stringent requirement on the inherent property, quality, and morphology of the antioxidation coating, an ultra-thin, single crystalline, epitaxial Au shell with atomic smooth surface and Ag-Au interface is an ideal choice. Being a noble metal, $\mathrm{Au}$ is more resistive to oxidation than other transition metals. Au is among the most conductive metals and is only second to $\mathrm{Ag}$ in terms of the high plasmonic enhancement and low plasmonic loss in the visible and near infrared (NIR) spectral range. Au also has a small lattice mismatch $(\sim 4 \%)$ to $\mathrm{Ag}$, making possible the epitaxial deposition of $\mathrm{Au}$ on the AgNW surface as a single crystalline, conformal coating with a smooth Ag-Au interface and external surface. However, achieving such an ideal coating condition is not trivial due to the galvanic replacement reaction between $\mathrm{Au}^{+}$ or $\mathrm{Au}^{3+}$ and metallic Ag, which results in the formation of hollow alloyed $\mathrm{Au}-\mathrm{Ag}$ nanostructures instead of the desired core-shell structure $[48,49]$. To solve this problem, strategies have been developed to suppress the galvanic replacement by complexing gold ions to lower the reduction potential of the dominant $\mathrm{Au}$ species. Galvanic replacement-free growth of an ultra-thin (2-3 atomic layers) gold shell on AgNWs was first demonstrated under the alkaline condition at $60^{\circ} \mathrm{C}$ that improves the chemical stability of AgNWs in $\mathrm{O}_{2}$ and oxidative solutions [43]. Recently, epitaxial growth of a thick Au coating $(\sim 35 \mathrm{~nm})$ on AgNWs was also realized via a sulfite-assisted protocol [44]. The coating was thick enough to offer good protection against ambient and strong oxidative environments. However, the thick and rough coating would increase the optical absorption and the haze factor [50], limiting the performance of the core-shell NW in transparent electrodes, and would also introduce significant optical loss. The precise control of the Au coating thickness and quality is the key to balance performance and stability in AgNW devices. Herein, we report the scalable, room-temperature solution-phase strategy to grow a conformal epitaxial $\mathrm{Au}$ shell on the AgNW surface with accurately controlled coating thickness for the optimization of chemical stability and material property. The conformal and high-quality coating provides long-term oxidation protection in various working environments, such as in air and solution, under heat, light, moisture, and mechanical stress. The as-synthesized Au@Ag core-shell NWs have atomically smooth surfaces and Ag-Au interfaces to minimize additional electron scattering and optical loss from the coating. It was demonstrated that various devices fabricated with the Ag@Au core-shell NWs, such as transparent electrode, plasmonic waveguide, nanofocusing probe, SERS substrate, and atomic force microscopy (AFM) probe, show strong performances comparable to those fabricated with pure AgNWs with significantly extended lifetime and stability. The strategy developed here is cost-efficient and compatible with large-scale device fabrication, offers a universal solution to increase the stability of various high-performance AgNW-based devices for commercialization.

\section{Experimental}

\subsection{Synthesis of AgNWs}

In a typical synthesis, $10 \mathrm{~mL}$ of ethylene glycol (EG) was added to a round-bottom flask $(50 \mathrm{~mL}$, equipped with a magnetic stirring bar); the flask was then suspended in an oil bath and heated at $170{ }^{\circ} \mathrm{C}$ for $15 \mathrm{~min}$. Then, $40 \mu \mathrm{L}$ of copper chloride/EG solution $\left(\mathrm{CuCl}_{2}, 0.0073 \mathrm{M}\right)$ was injected into this $10 \mathrm{~mL}$ of EG solution. After $15 \mathrm{~min}, 10 \mathrm{~mL}$ of $\mathrm{AgNO}_{3}$ solution $(0.15 \mathrm{M}$, in EG, freezing for $10 \mathrm{~min}$ before use) and $10 \mathrm{~mL}$ of PVP solution $(0.45 \mathrm{M}$ as calculated in terms of the repeating unit, in EG) were added dropwise to the heated EG solution at a pace of $150 \mu \mathrm{L} / \mathrm{min}$. The growth of nanowires was monitored by sampling small portions of the reaction mixture for UV-vis measurement every $10 \mathrm{~min}$. Mild stirring with $260 \mathrm{rpm}$ was maintained throughout the whole process. Finally, AgNWs were collected by centrifugation and washed with ethanol four times.

\subsection{Synthesis of Ag@Au core-shell NWs}

Preparation of Au growth solution: $40 \mu \mathrm{L}$ of $\mathrm{HAuCl}_{4}(0.01 \mathrm{M})$, $240 \mu \mathrm{L}$ of $\mathrm{NaOH}(0.2 \mathrm{M}), 3 \mathrm{~mL}$ of $\mathrm{Na}_{2} \mathrm{SO}_{3}(0.02 \mathrm{M})$ were added to $4.72 \mathrm{~mL}$ of $\mathrm{H}_{2} \mathrm{O}$ sequentially. The solution was left undisturbed for at least 10 h. Preparation of Ag@Au core-shell NWs: In a typical synthesis, $1 \mathrm{~mL}$ of the above solution of AgNWs were dispersed into $2.55 \mathrm{~mL}$ of $\mathrm{H}_{2} \mathrm{O}$, then $2 \mathrm{~mL}$ of $2 \%$ PVP aqueous solution, $240 \mu \mathrm{L}$ of AA $(0.5 \mathrm{M})$ and $50 \mu \mathrm{L}$ of diethylamine were added to $20 \mathrm{~mL}$ glass vial, respectively. Noting that a small amount of diethylamine could help eliminate the energy difference for deposition between each different facet of silver as diethylamine is known to be unselectively adsorbed on the surface of AgNWs without causing etching. A homogeneous $\mathrm{Au}$ deposition on the AgNW surface might be achieved [51-53]. Then using a syringe pump, the as-prepared Au growth solution was slowly injected into the solution of AgNWs at a rate of $0.05 \mathrm{~mL} / \mathrm{min}$ to deposit a thin layer of $\mathrm{Au}$ on the $\mathrm{AgNW}$ 
surface. The Au coating thickness could be tuned by controlling the reaction time. The as-prepared Ag@Au NWs were collected by centrifugation, washed with $\mathrm{H}_{2} \mathrm{O}$ twice, and stored in $\mathrm{H}_{2} \mathrm{O}$ for further use.

\subsection{Fabrication of NW-based transparent electrodes}

Nanowires (AgNWs or Ag@Au core-shell nanowires) were diluted with toluene and sonicated for $10 \mathrm{~min}$ to form a homogeneous solution. The transparency film was prepared by filtering the nanowire solution onto a nitrocellulose porous membrane with a $220-\mathrm{nm}$ pore size via vacuum filtration. The nanowire network was then entirely transferred onto a transparent substrate (PET, glass, or PDMS) by applying pressure to the backside of the membrane.

\subsection{Synthesis of NW-based AFM probe}

Based on our previous method [15], The AgAuNW-AFM probe was fabricated by attaching a single Ag@Au core-shell nanowire onto the pyramidical tip's sidewall of a commercial AFM cantilever by using a benchtop manipulation procedure. First, Ag@Au core-shell nanowires were dispersed onto a clean PDMS substrate and selected with an upright optical microscope imaging system to pick up an appropriate Ag@Au core-shell nanowire. A tungsten probe mounted on a motorized four-axis micromanipulator (MP-285, Sutter Instrument, $40 \mathrm{~nm}$ resolution) was used to get the target nanowire and place it in the desired position on the pyramidical tip, and finely adjust the protruding length by gently pushing along the nanowire. After the Ag@Au core-shell nanowire had been mounted, another tungsten probe was used to apply a small amount of epoxy glue on the Ag@Au core-shell nanowires-cantilever junction to enhance the adhesion and prevent the detachment of the Ag@Au core-shell nanowire during the tapping-mode measurement.

\subsection{Sheet resistance measurement}

The sheet resistance measurement was modified based on Hu's work [54]. A glass substrate $(25 \mathrm{~mm} \times 25 \mathrm{~mm} \times 0.1 \mathrm{~mm}$, Fischer Scientific) was rinsed with acetone and methanol at least three times, then $4 \mathrm{~nm} / 100 \mathrm{~nm}(\mathrm{Cr} / \mathrm{Au})$ was deposited via a custom Angstrom Engineering thermal evaporator system. An AgNW and Ag@Au core-shell nanowire-based transparent electrode was fabricated via vacuum filtration. The transparent electrodes were then transferred onto the prepared mask separately. Finally, the sheet resistance of both electrodes was measured using a Signatone CM200 probe station and Agilent 4155A Semiconductor Parameter Analyzer. For the humidityassisted oxidative acceleration test, both AgNW and Ag@Au core-shell nanowire-based transparent electrodes were put into a tin container that stayed inside a larger aluminum can, which was filled with $300 \mathrm{~mL}$ of water at the bottom to imitate an environment with $100 \%$ humidity. The can was placed inside a furnace (Thermo Scientific) at $80^{\circ} \mathrm{C}$.

\subsection{Characterization}

The transmission electron microscopy (TEM) images were taken with a Tecnai 12 transmission electron microscopy operating at $120 \mathrm{kV}$. UV-Vis-Near IR (NIR) spectra were measured with Varian Cary $50 \mathrm{UV} / \mathrm{Vis}$ spectrophotometers 190-1,100 nm. Atomic force microscopy (AFM) measurements were carried out on an AIS-NT AFM and were operated in non-contact mode. Scanning electron microscopy (SEM) was conducted on a NovaNanoSEM 450. Photoluminescence images and spectra of Ag@Au core-shell nanowires (or AgNWs) were obtained using a Horiba LabRam Spectrophotometer.

\section{Results and discussion}

Figure 1(a) shows a schematic of the synthetic route of the epitaxial Ag@Au NWs. The AgNWs used in this work were synthesized via a modified mediated polyol-mediated process and purified for further use (see Materials and Methods section). To achieve galvanic replacement-free deposition of the $\mathrm{Au}$ shell on the AgNW surface, the reduction potential of the gold ion source was reduced through complexing the $\mathrm{Au}^{3+}$ ions in the $\mathrm{HAuCl}_{4}$ precursor solution with sulfite $\left(\mathrm{SO}_{3}{ }^{2-}\right)$ to form a highly stable complex $\mathrm{Au}\left(\mathrm{SO}_{3}\right)_{2}{ }^{2-}$. With a $\mathrm{p} K_{\mathrm{sp}}$ of -26.8 , the $\mathrm{Au}\left(\mathrm{SO}_{3}\right)_{2}{ }^{2-}$ complex has high stability, resulting in a much lower reduction potential $\left(E^{0}=0.111 \mathrm{~V}\right)$ than that of $\mathrm{Ag}^{+}\left(E^{0}=\right.$ $0.8 \mathrm{~V}$ ) [55]. In addition, a strong reducing agent (i.e., ascorbic acid, AA) was used to further suppress the oxidative etching of the AgNW surface by the $\mathrm{Au}^{3+}$ ions [56]. The gold-sulfite complex solution was slowly injected via a syringe pump into the reaction solution containing the reducing agent $\mathrm{AA}$ and AgNWs under vigorous stirring to control the Au precursor concentration and homogeneity. To achieve the high-quality, etch-free $\mathrm{Au}$ deposition on the AgNW surface, we first investigated the effect of $\mathrm{Na}_{2} \mathrm{SO}_{3}: \mathrm{HAuCl}_{4}$ feeding ratios in synthesizing high-quality Ag@Au core-shell nanowires. As (a)

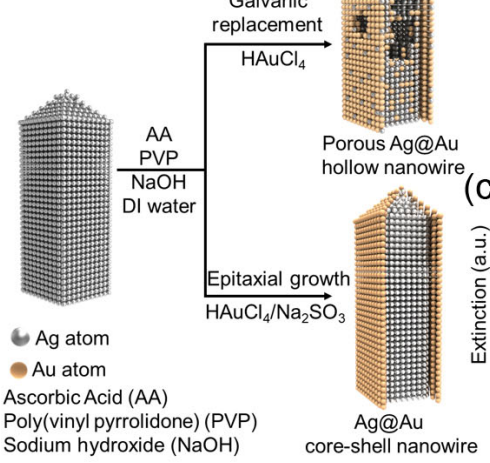

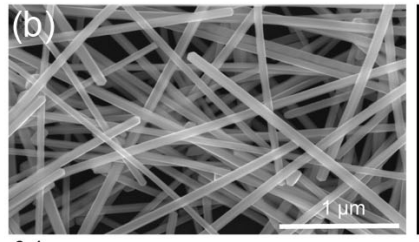
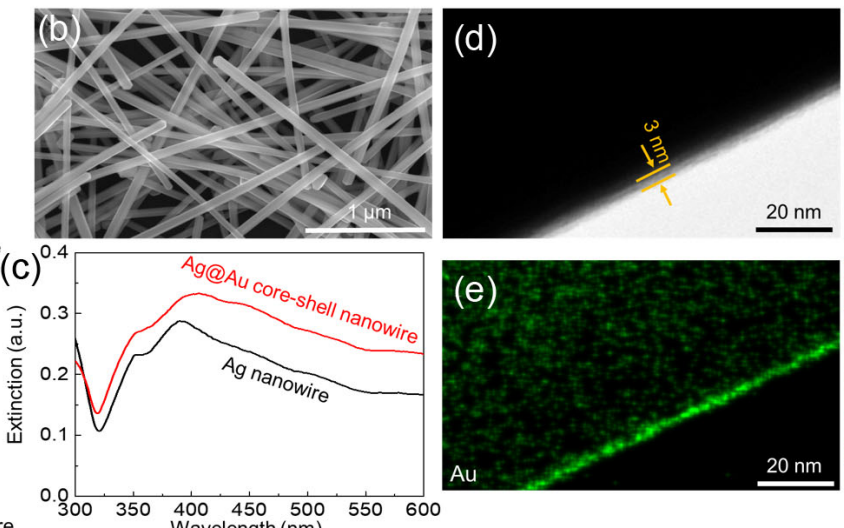

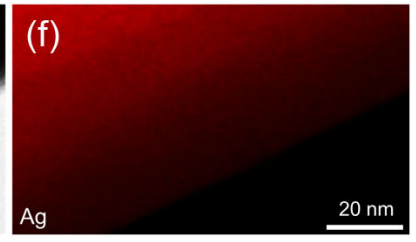

(g)

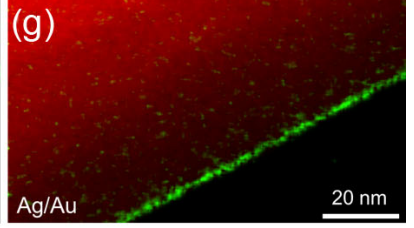

Figure 1 Synthesis of the Ag@Au core-shell NWs. (a) Schematic illustration of the reduction of $\mathrm{HAuCl}_{4}$ in the presence of $\mathrm{AgNW}_{\mathrm{N}} \mathrm{with} /$ without $\mathrm{Na}_{2} \mathrm{SO}_{3}$. The etch-free growth of a smooth epitaxial Au shell on the AgNW surface relies on complexing Au ions with $\mathrm{SO}_{3}{ }^{2-}$ that lowers the reduction potential of the Au precursor species. (b) SEM image of Ag@Au core-shell NWs. (c) UV-Vis extinction spectrum of AgNW before (black curve) and after (red curve) 3-nm Au shell deposition. (d) TEM image of a Ag@Au core-shell NW ( 3 nm Au). (e)-(g) Elemental mapping of Ag and Au from Ag@Au core-shell NW $(\sim 3 \mathrm{~nm} \mathrm{Au})$. The red color indicates Ag, whereas the green color indicates Au. 
shown in Fig. S1(a) in the Electronic Supplementary Material (ESM), rough core-shell NW surface and significant etching were observed with a low sulfite to gold ratio (2:1). As the ratio was reduced, the NW surface becomes smooth, but still showing significant etching on the NW tips and along the ridges of penta-twinned AgNWs until a sulfite to gold molar ratio of 150:1 (Figs. S1(b)-S1(e) in the ESM). This result shows a high tendency of local oxidative etching of free $\mathrm{Au}^{3+}$ ions to the AgNW surface even with the complexing agent. And an extremely low free $\mathrm{Au}^{3+}$ concentration must be maintained by adding $\mathrm{SO}_{3}{ }^{2-}$ in large excess. Based on this result, subsequent synthesis of the Ag@Au core-shell nanowires were optimized with a $\mathrm{Na}_{2} \mathrm{SO}_{3}: \mathrm{HuCl}_{4}$ feeding ratios of $>150: 1$ to completely suppress the oxidative etching of the AgNW surface.

The SEM image (Fig. 1(b)) of the Ag@Au core-shell nanowires obtained from the optimized synthesis approach shows an overview of NWs (average diameter of $250 \mathrm{~nm}$ ) with uniform and smooth surfaces without signs of etching. The TEM image in Fig. 1(d) shows a uniform $3 \mathrm{~nm}$ Au shell with a smooth surface and Au-Ag interface. The energy-dispersive X-ray spectroscopy (EDS, Figs. 1(e)-1(g)) mapping from the same region also demonstrated a well-defined core-shell structure with a Ag (red color) core and a uniform thin Au (green color) shell. The shell thickness is consistent with that measured in the TEM image (Fig. 1(d)). UV-Vis extinction spectrum of Ag@Au core-shell NWs with a 3 nm shell thickness (Fig. 1(c)) shows a very slight increase in absorption and a slight red shift of absorption peaks as compared with pristine AgNW, consistent with what was observed in other Ag@Au core-shell nanostructures $[43,55]$, however, due to the thin Au coating compared to the AgNW diameter, the changes in the absorption intensity and frequencies are very small.

The Au shell thickness can be accurately controlled by varying the amount of gold-sulfite complex injected into the reaction solution, which can be adjusted by changing the reaction time. The UV-Vis spectroscopy (Fig. 2(a)) shows a progressive redshift in the extinction peak position and a continuous increase in intensity as the reaction time increases and more $\mathrm{Au}$ precursor was added, consistent with growing $\mathrm{Au}$ shell thickness $[43,55]$. This finding also confirmed the absence of galvanic replacement or oxidative etching, which is typically accompanied by an apparent attenuation of the original surface plasmon resonance peaks of Ag nanostructures [49, 57]. It is also worth noting that even with the longest reaction time, there is no sign of nucleation of separate $\mathrm{Au}$ nanoparticles, whose plasmonic peaks are typically observed at $\sim 520 \mathrm{~nm}$. The corresponding high-resolution TEM images (Figs. 2(b), 2(d), and 2(e)) of the Ag@Au core-shell nanowires synthesized with reaction times of 60,90 , and 120 mins, shows a coating thickness of $6 \mathrm{~nm}$ (Fig. 2(b)), $10 \mathrm{~nm}$ (Fig. 2(d)), and $15 \mathrm{~nm}$ (Fig. 2(e)), respectively. The TEM image of the tip of the Ag@Au core-shell NW (Fig. S2 in the ESM) further demonstrated a smooth and uniform coating on the NW tip with the coating thickness consistent with the side walls. The measured thickness is based on the contrast between the Ag core and Au shell in the TEM images, which is proven consistent with the EDS elemental mapping (e.g., Figs. 1(d), 1(e), 2(b), and Fig. S3 in the ESM). For all shell thicknesses, the Au coatings are uniform and smooth with a clean and etch-free interface between $\mathrm{Au}$ and Ag. Figure 2(c) is the high-resolution TEM (HRTEM) image of the region marked with the red box in Fig. 2(b), showing the sub-nm level smoothness of the Au layer. The continuous lattice fringes from the Ag core to the $\mathrm{Au}$ shell manifested unambiguously the epitaxial growth and the single crystalline nature of the Au shell, which is critical for maintaining the electrical/optical properties of AgNWs and optimizing chemical stability of the core-shell nanowires due to the minimized interfacial defects.

The chemical stability of the Ag@Au core-shell nanowires was evaluated by benchmarking against the pure AgNWs under different conditions. The results suggest that a $3 \mathrm{~nm}$ Au shell can effectively serve as an antioxidation barrier of the AgNWs. When exposed to air at room temperature, the Ag@Au core-shell NWs remained stable against environmental oxidation for as long as six months, as evidenced by the smooth surface in the SEM images taken at day 0 and day 183 (Figs. 3(a) and 3(b)). In contrast, pristine AgNWs deteriorate fast under ambient conditions. After only ten days, the wire surfaces were covered with silver oxide nanoparticles (Figs. 3(c) and 3(d)), typical of severely oxidized Ag nanostructures. The 3-nm high-quality epitaxial $\mathrm{Au}$ shell was even able to protect the AgNW from strong oxidants such as hydrogen peroxide $\mathrm{H}_{2} \mathrm{O}_{2}$. After overnight treatment of $\mathrm{H}_{2} \mathrm{O}_{2}$ (2 wt.\%) aqueous solutions, the Ag@Au core-shell NWs were still intact as shown in the before and after SEM images of the sample (Fig. 3(e), insets), as well as the UV-Vis spectra (Fig. 3(e), top) showing no obvious sign of attenuation of the plasmonic band due to etching and oxidation of the AgNW.

Biosensing applications typically require long-term stability in physiological solutions. The Ag@Au core-shell NWs remained stable in phosphate-buffered saline (PBS) solution for over 21 days, showing no noticeable spectral changes in the UV-Vis spectrum of the sample (Fig. S4(a) in the ESM). The SEM image of the sample after 21 days of soaking in the PBS solution
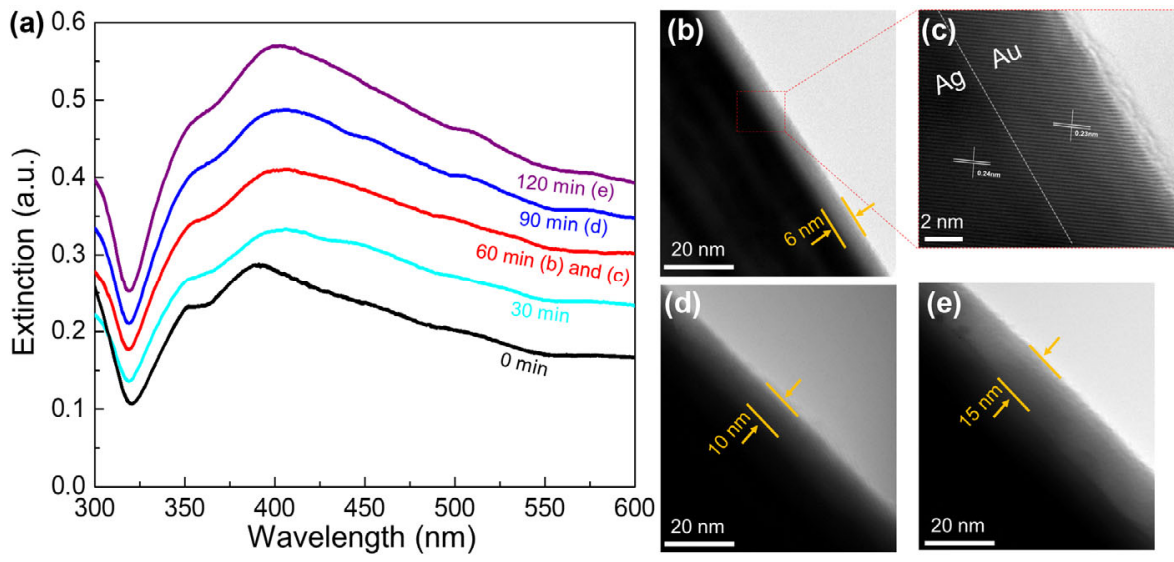

Figure 2 Tunable growth of the Au shell on AgNW surfaces. (a) UV-Vis extinction spectrum of Ag@Au core-shell NWs with varied reaction time; (b) and (c) TEM image of Ag@Au core-shell NW ( 6 nm Au) and corresponding high-resolution TEM image to show the epitaxial growth of Au on AgNW; (d) and (e) TEM image of Ag@Au core-shell NW with thicker Au shell ( $10 \mathrm{~nm} \mathrm{Au} \mathrm{(d);15} \mathrm{nm} \mathrm{Au} \mathrm{(e)).}$ 

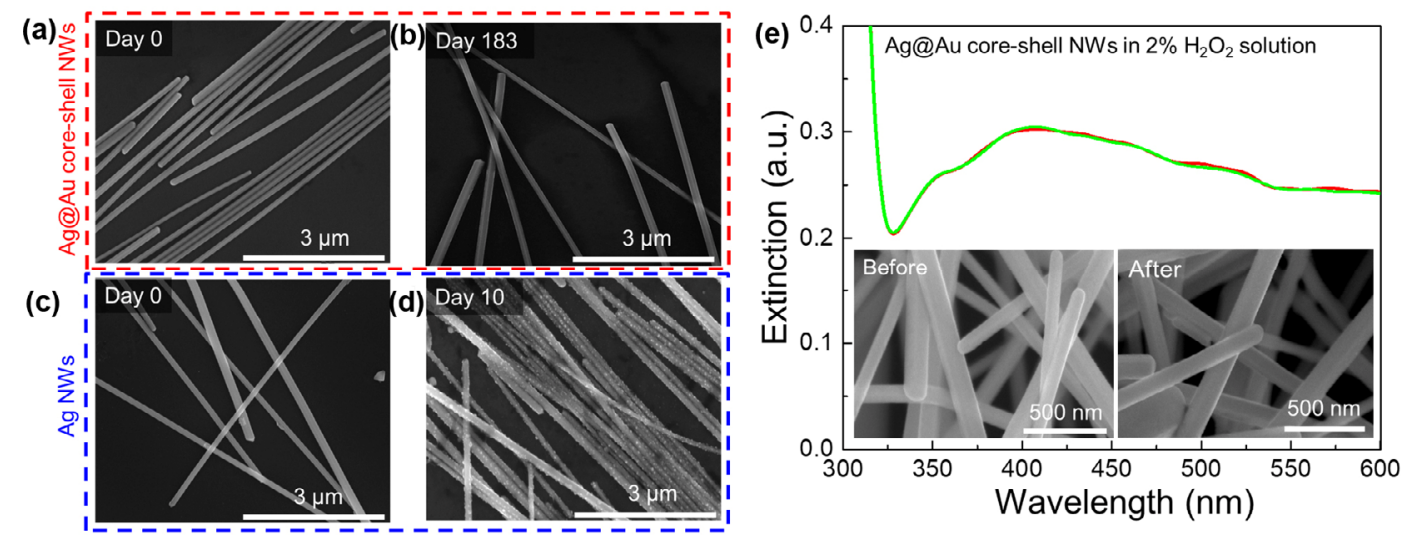

Figure 3 Chemical stability of Ag@Au core-shell NWs ( $3 \mathrm{~nm} \mathrm{Au})$ upon exposure to air and hydrogen peroxide aqueous solution (2 wt.\%). (a) and (b) SEM images of the Ag@Au core-shell NWs after exposure to air for 0,183 days, respectively. (c) and (d) SEM images of pristine AgNWs after exposure to air for 0, 10 days, respectively. (e) The UV-vis extinction spectrum of Ag@Au core-shell NWs before (red curve) and after (green curve) treatment with $\mathrm{H}_{2} \mathrm{O}_{2}$ solution overnight. The inset is the corresponding SEM images of Ag@Au core-shell NWs before (left) and after (right) the $\mathrm{H}_{2} \mathrm{O}_{2}$ treatment.

also shows smooth surfaces without signs of oxidation (Fig. S4(a) in the ESM, inset). In contrast, pristine AgNWs underwent severe degradation in PBS solution in days, as evidenced by a significant decrease in UV-Vis absorption intensity as early as day 7 and followed by the complete disappearance of the signature AgNW plasmonic bands within 21 days (Fig. S4(b) in the ESM). Therefore, the 3-nm high-quality epitaxial Au shell can effectively preserve the plasmonic/optical performance of AgNWs for bio and chemical sensing applications performed in aqueous solutions, even in the presence of salt and oxidative chemicals.

We further evaluated the performance and stability of transparent mesh electrodes fabricated with the Ag@Au core-shell NWs on a flexible polyethylene terephthalate (PET) substrate via a simple vacuum filtration method [58]. A dilute nanowire suspension was vacuum filtered through filter paper. The resulting NW filtration mesh was then transferred onto a flexible PET substrate by pressing the filter paper against the substrate to form the NW mesh electrode. Figure 4(a) shows the transmittance spectra (400-800 nm) and the corresponding sheet resistance of Ag@Au core-shell NW mesh electrode with different wire loading densities in the range of $75 \%$ to $95 \%$ transmittance benchmarked at $550 \mathrm{~nm}$. The corresponding optical (top panels) and SEM (bottom panels) images of the NW mesh electrodes are shown in Fig. 4(b). The film sheet resistance is as low as $4.67 \Omega /$ squnder $75 \%$ optical transmittance. With increasing optical transmittance and decreasing wire density, the film sheet resistance gradually increased to $9.88 \Omega / \mathrm{sq}$ at $85 \%, 17.52 \Omega /$ sq at $90 \%$, and eventually $37.41 \Omega /$ sq at $95 \%$ transmittance. The performance stands out in comparison with other published work on different materials candidates for flexible electrodes (Fig. 4(c)), such as AgNW [59], copper nanowire [59], carbon nanotube (CNT) [60], graphene [59], and PEDOT $[24,61]$, that demonstrate $17.3 \Omega / \mathrm{sq}$ at $91 \%$, $62.5 \Omega /$ sq at $90 \%, 71.05 \Omega /$ sq at $92 \%, 87.5 \Omega /$ sq at $93 \%$, and $100 \Omega /$ sq at $91 \%$, respectively. In particular, the Ag@Au core-shell NW transparent electrodes have demonstrated performance comparable to commercial indium tin oxide (ITO) performance
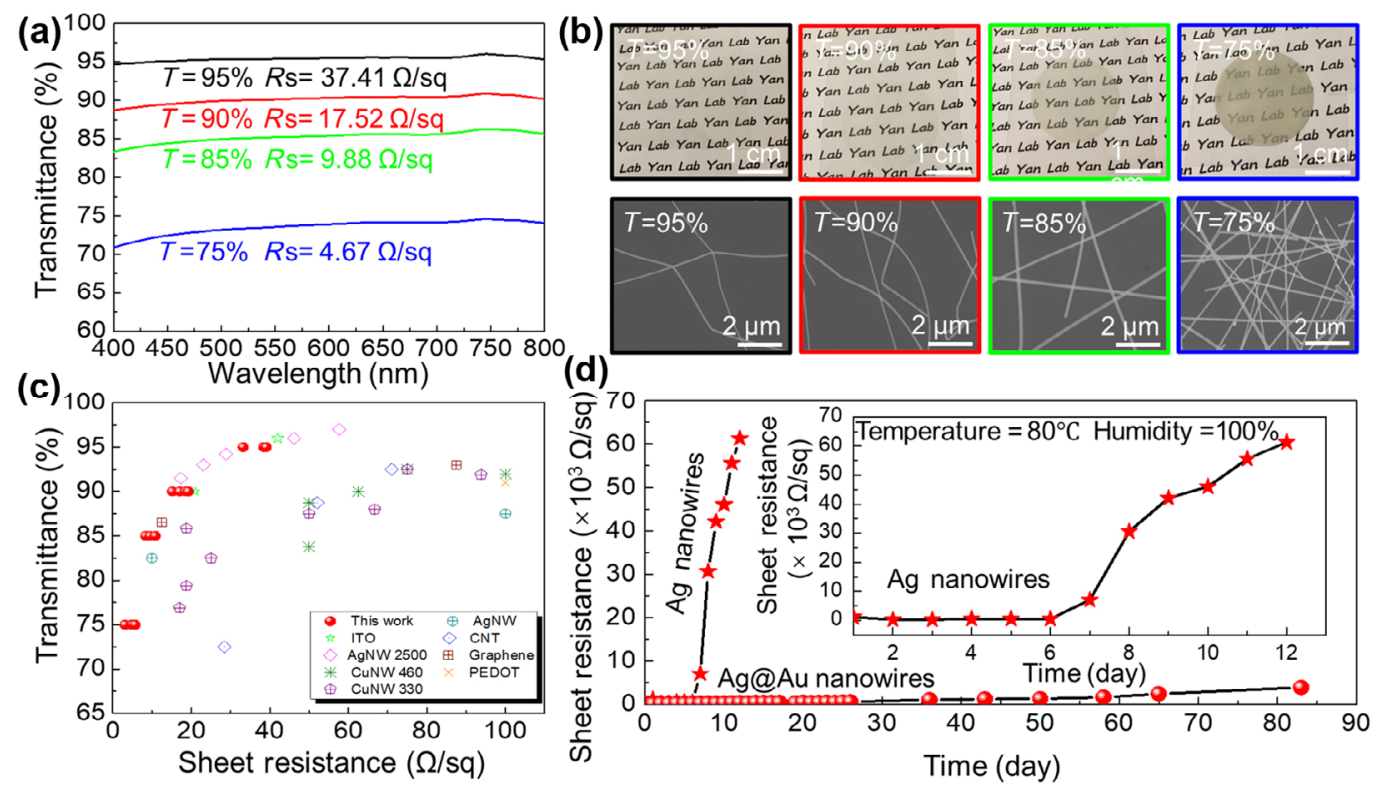

Figure 4 Ag@Au core-shell NW transparent electrodes and their optical and electrical performance. (a) and (b) Wavelength-dependent optical transmission, electrical conductance, and corresponding SEM images and optical images of transparent conductive films with increasing Ag@Au core-shell NW loading (from left to right). (c) Plot of optical transmittance vs. sheet resistance of Ag@Au core-shell NW conductive films (solid red dots). For comparison, published data of the current state-of-the-art transparent conductors, including ITO, CNT, PEDOT, AgNW, and Cu nanowire were also plotted. (d) Long-term stability of Ag@Au core-shell NW ( $3 \mathrm{~nm} \mathrm{Au}$ ) and AgNW transparent electrodes in a high temperature (80 $\left.{ }^{\circ} \mathrm{C}\right)$ and high humidity (100\%) environment. 
(21 $\Omega /$ sq at $90 \%$ [62]) and pristine AgNW [59] (17.3 $\Omega /$ sq at $91 \%)$, which is so far the best candidate for flexible transparent electrodes. The stability of the Ag@Au core-shell NW mesh electrode is tested in an accelerated aging condition under heat $\left(80{ }^{\circ} \mathrm{C}\right)$ and moisture (100\% humidity). No significant change in the sheet resistance was found for as long as 84 days (Fig. 4(d)). In contrast, the sheet resistance of pristine AgNW mesh electrode showed a sharp increase of $5,000 \%$ after the first week of exposure to the hot $\left(80{ }^{\circ} \mathrm{C}\right)$ and humid $(100 \%)$ environments and a complete failure after 12 days (Fig. 4(d), inset).

AgNWs are capable of confining light to subwavelength dimensions in the form of surface plasmon polaritons (SPPs), which are electron density wave that travels along with the metal-dielectric interface [7]. Plasmonic waveguides that can propagate SPPs represent one of the most promising structures to beat the diffraction limit imposed by conventional dielectric optics. Chemically synthesized Ag nanowires have demonstrated great promise for plasmonic-photonic integrated circuits and other subwavelength optical devices due to their very low SPP propagation losses resulting from the inherent high conductivity of $\mathrm{Ag}$ and the high crystallinity and smooth surface of the NWs [7]. However, the growth of silver oxide particles on the AgNW surface due to oxidization could significantly amplify plasmonic losses originating from radiative scattering due to increased surface roughness and nonradiative decay from Joule heating and eventually leads to device failure [13]. Although the $3 \mathrm{~nm} \mathrm{Au}$ shell offers good protection against oxidation in the air that can prolong the lifetime of AgNW plasmonic waveguides, a major concern is the additional propagation loss introduced by the Au shell. The increase in loss can be detrimental with a rough and/or thick Au coating, but even with a thin, smooth epitaxial coating, the impact still needs to be evaluated due to the inherently lower conductivity of $\mathrm{Au}$ compared to Ag. Following the method previous established in our group $[63,64]$, we estimated the plasmon propagation length $(L)$, the characteristic length after which the SPP intensity decays by a factor of $1 / e$, and the measure of the propagation loss at a given frequency, of AgNWs with and without the 3-nm Au coating. Figure 5(a) is a dark-field optical image of the typical experimental setup, where a tapered single-mode optical fiber tip was placed in contact with the suspended portion of the NW placed on the edge of a PDMS substrate. Lasers of different frequencies are coupled into single-mode optical fiber and delivered to the junction between the tapered fiber tip and the NW to excite the SPPs in the NW. Figure 5(b) is the corresponding waveguiding image with the microscope illumination turned off and the laser light introduced from the optical fiber stays on. Without the microscope illumination, the profiles of the optical fiber and the NW are no longer visible. The big bright spot at the fiber-NW junction is the scattered laser that excites the SPP in the AgNW. The faint spot (Fig. 5(b), inset) at the tip of the NW originates from the SPPs traveling in the AgNW that scattered back to the free space at the tip, indicating the successful excitation of the SPP. The optical fiber is mounted on a micromanipulator and can be moved up and down the NW to change the distance between the junction and the NW tip. Assuming a stable excitation efficiency along the NW, a shorter propagation distance will lead to less attenuation and a brighter tip scattering spot. By correlating the two, the propagation length can be calculated [64].

Figures 5(c) and 5(d) plot the measured tip emission intensity of a Ag@Au core-shell NW (red curve) and a AgNW (green curve) as a function of the propagation distance under 671 and $532 \mathrm{~nm}$ excitations, respectively. Both wires are around (a)

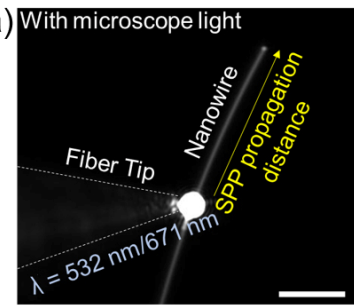

(c) $\quad \alpha=11.03071 \mathrm{~nm}$

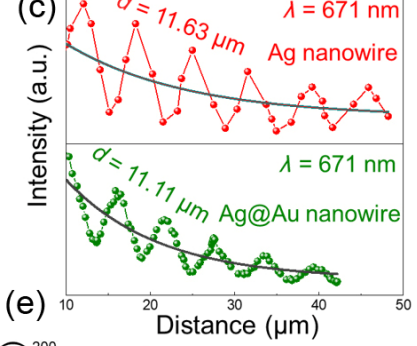

$\widehat{\text { E. }}_{160-}^{200} \quad 1=671 \mathrm{~nm}$

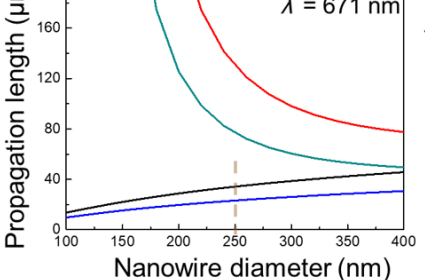

(b) Without microscope light
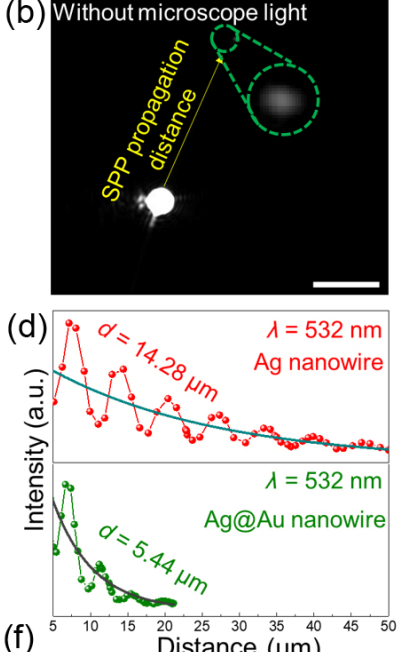

(f)

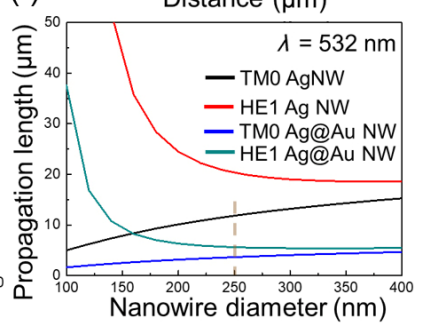

(d) if $\alpha=\quad \lambda=532 \mathrm{~nm}$

Figure 5 Measurement of the SPP propagation length in a free-standing nanowire (AgNW or Ag@Au core-shell NW ( $3 \mathrm{~nm} \mathrm{Au})$ ). (a) Dark-field optical image (microscope light on, laser on) of the measurement setup. A target nanowire was placed on the edge of a PDMS substrate. The tapered tip of a laser-coupled optical fiber was put into physical contact with the nanowire to excite its SPPs. The SPPs propagated along the nanowire to its distal end, where it was scattered back into the free space. The far-field emission at the suspended AgNW tip was collected as the optical fiber tip was slid along the nanowire. (b) Waveguide images (microscope light off, laser on) of the nanowire at specific SPP propagation distances. Insets: enlarged images of the tip emission spot. (c) and (d) Tip emission intensity measured as a function of propagation distance at 671 and $532 \mathrm{~nm}$ excitation wavelengths. (e) and (f) Numerical simulation of the propagation length as a function of nanowire diameter at $671 \mathrm{~nm}$ (e) and $532 \mathrm{~nm}$ (f) excitation wavelengths. All scale bars are $10 \mu \mathrm{m}$.

$250 \mathrm{~nm}$ in diameter. As expected, the emission intensities of both wires show a general decaying trend as the propagation distance increases due to the increased propagation losses, while the intensity oscillations originate from the beating of different SPP modes [64]. The Ag@Au core-shell NW shows a slight reduction in propagation length $\left(L_{\mathrm{Ag} @ A u, 671 \mathrm{~nm}}=11.11 \mu \mathrm{m}\right)$ as that of the pristine $\operatorname{AgNW}\left(L_{\mathrm{Ag}, 67 \mathrm{~nm}}=16.63 \mu \mathrm{m}\right)$ under $671 \mathrm{~nm}$ excitation. However, the Ag@Au core-shell NW does show more significant intensity decay than pristine AgNW under $532 \mathrm{~nm}$ excitation (Ag@Au core-shell nanowire: $L_{\mathrm{Agg} @ \mathrm{Au}, 532 \mathrm{~nm}}=$ $5.44 \mu \mathrm{m}$; AgNW: $L_{\mathrm{Ag}, 532 \mathrm{~nm}}=18.08 \mu \mathrm{m}$ ), due to the stronger absorption of $\mathrm{Au}$ in the green region. This observed trend is consistent with the numerical simulation result. Figures 5(e) and 5(f) show calculated propagation lengths of the two co-existing SPP modes ( $\mathrm{TM}_{0}$ and $\left.\mathrm{HE}_{1}\right)$ of both Ag@Au core-shell and pristine $\mathrm{Ag}$ nanowire in a diameter range from 100 to $400 \mathrm{~nm}$. At a diameter of $250 \mathrm{~nm}$ (marked by the dash lines in Figs. 5(e) and 5(f)), both SPP modes of Ag@Au core-shell nanowire shows a more significant decrease under $532 \mathrm{~nm}$ excitation (Fig. 5(f)) than under $671 \mathrm{~nm}$ excitation (Fig. 5(e)) compared to pristine AgNW, in good agreement with the trend observed in the experimental results.

Despite the frequency-dependent increase in loss, the Ag@Au core-shell NW can still function as high-performance plasmonic 
components, even under $532 \mathrm{~nm}$ excitation, especially those that do not require long propagation distances and more loss tolerant. Figure 6 compares the optical performance and stability of nanofocusing probes fabricated from pristine and Au-coated AgNWs for chemical nanoscopy [13]. As shown in the darkfield optical images and schematics (Figs. 6(a) and 6(f)), the probe is constructed by putting a Ag or core-shell nanowire in physical contact with a laser-coupled tapered optical fiber tip. The laser is optically coupled from the tapered fiber to the SPPs in the nanowire through mode-matching [13]. Figures 6(b) and $6(\mathrm{~g})$ are the optical image of the same view as in Figs. 6(a) and $6(\mathrm{f})$, but with the laser turned on and the microscope illumination off. Both pristine and Au coated AgNWs probes show high-efficiency optical coupling between the fiber and the nanowire, characterized by the bright emission at the NW tips and negligible scattering from the fiber-wire junction $[13,65]$. The performance of the Ag@Au core-shell nanowire stays stable even after 21 days (Figs. 6(h) $-6(\mathrm{j})$ ), indicating the robust protection of the 3-nm epitaxial Au shell against oxidation under optical stress for optical/plasmonic applications. In contrast, scattering spots start to show along the pristine AgNWs due to the growth of oxide nanoparticles (as visualized in Fig. 3(d)) within a week, resulting in a decreased light-delivery efficiency to the NW tip and eventually device failure (Figs. 6(c)-6(e)).

In addition to the SPP characterization, surface plasmon resonance (SPR) is another essential characterization technique for AgNW based plasmonic devices intended for SERS applications, which enable label-free, nondestructive, highsensitivity chemical analysis $[66,67]$. One concern of using Ag@Au core-shell nanowires in SERS sensing applications is whether the Au shell will introduce extra background due to its fluorescence under $532 \mathrm{~nm}$ excitation, the most popular SERS excitation wavelength. Here we compared the SERS spectra of a self-assembled monolayer of 4-aminothiopenol (4-ATP) from a single Ag@Au core-shell nanowire vs. a single pristine Ag NW using a confocal Raman microscopy that allows for sampling from a similar length from both wires (Fig. S5 in the ESM). Since the diameters of the two nanowires are also similar, the total metal surface area that offers SERS enhancement under laser excitation is relatively comparable. Assuming the same packing density of the 4-ATP molecule in the self-assembled monolayer, given the similar surface chemistry and atomic density of the Au and Ag surface, the measured SERS intensity can then be directly compared to qualitatively compare the SERS activity of the two types of wires. From the two spectra in Fig. S5 in the ESM, it can be concluded that the Ag@Au core-shell and pristine Ag NW offer a similar level of signal enhancement, and no significant increase in background fluorescence was observed from the Ag@Au core-shell nanowire, thanks to the ultra-thin Au coating $(\sim 3 \mathrm{~nm})$. Considering the demonstrated stability of the Ag@Au core-shell nanowires in air and solution, they would constitute a high-performance, air- and solution-stable SERS platform for chemical and biological analysis.

The stability of Ag@Au core-shell nanowires under mechanical stress was evaluated with AgNW-based AFM probes [15]. AgNW-based SPM probes, including high-aspect-ratio AFM probe, AFM and STM-based TERS probes, chemical nanoscopy probes $\left[11,13^{-15}\right]$, bears enormous potential in changing the landscape of commercial SPM probes due to their outstanding performance and unique applications. However, a remaining challenge hindering their commercialization is the limited shelf-life and device stability due to their vulnerability to oxidation under storage and working conditions, especially for tapping-mode AFM probes that work under constant mechanical stress. To evaluated the performance and stability of Ag@Au core-shell NW-based high-resolution, high-aspect-ratio AFM probes (AgAuNW-AFM) under tapping mode operation, AFM probes with pristine and $\mathrm{Au}$ coated AgNWs were fabricated following a previously established procedure [15]. As shown in the SEM image (Fig. 7(a)), the nanowire was mounted onto a commercial AFM cantilever's pyramidical tip using a customized 3D benchtop manipulator (see materials and methods section). High-quality tapping-mode AFM images were obtained on a test sample $(40-\mathrm{nm}$ Au film on E-beam evaporated to a clean silicon dioxide substrate) with both pristine and gold-coated AgNW probes with similar resolutions when they were freshly prepared (Figs. 7(b) and 7(c), top panel). While the AgNW-AFM probe experienced a gradual decrease in imaging resolution (Day 7 and Day 14, Fig. S6 in the ESM) and a sudden mechanical failure at Day 21 (Fig. 7(c), bottom panel), the AgAuNW-AFM probe showed robust imaging
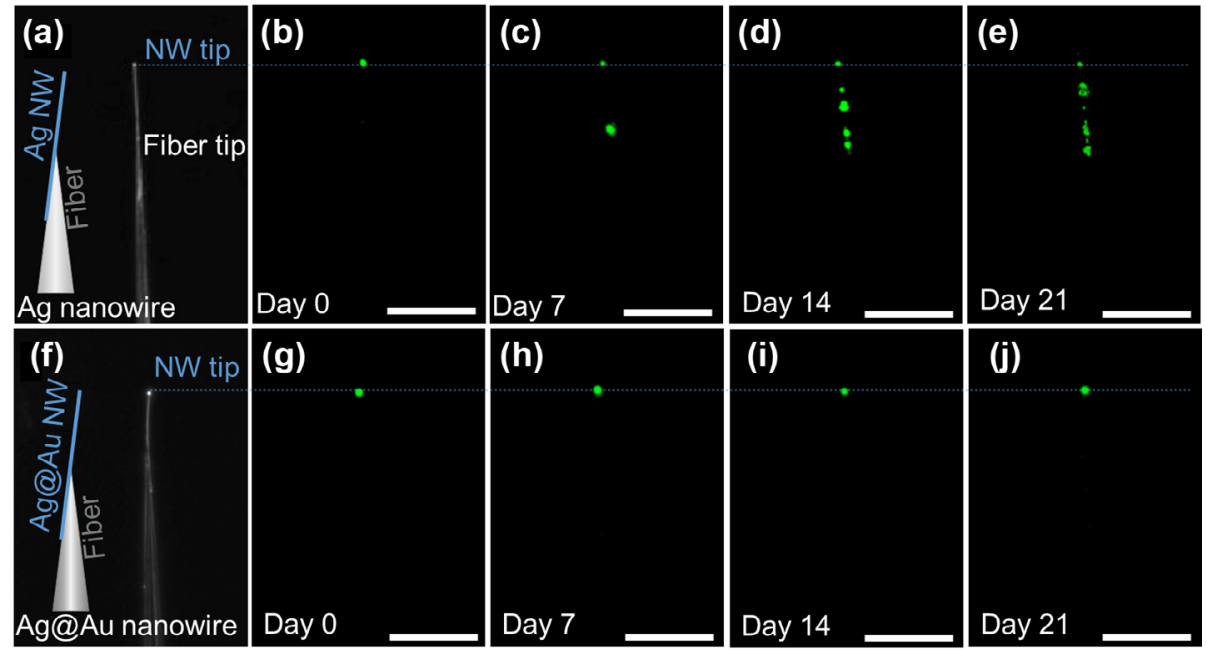

Figure 6 Stability of nanofocusing probes fabricated from AgNW and Ag@Au core-shell NW ( $3 \mathrm{~nm} \mathrm{Au}$ ). (a) Darkfield optical image of nanofocusing probe fabricated with pristine AgNW. Inset: schematic illustrating the device structure. The laser is coupled through the tapered tip of the optical fiber to launch SPPs into the AgNW. (b)-(e) Waveguide image (microscope light off, laser on) of the AgNW nanofocusing probe upon exposure to air for 0, 7, 14, 21 days. (f) Darkfield optical image of nanofocusing probe fabricated with Ag@Au core-shell NW. Inset: schematic illustrating the device structure. The laser is coupled through the tapered tip of the optical fiber to launch SPPs into the Ag@Au core-shell NW. (g)-(j) Waveguide image (microscope light off, laser on) of the Ag@Au core-shell nanowire after exposure to air for 0, 7, 14, 21 days, respectively. All scale bars are $40 \mu \mathrm{m}$. 


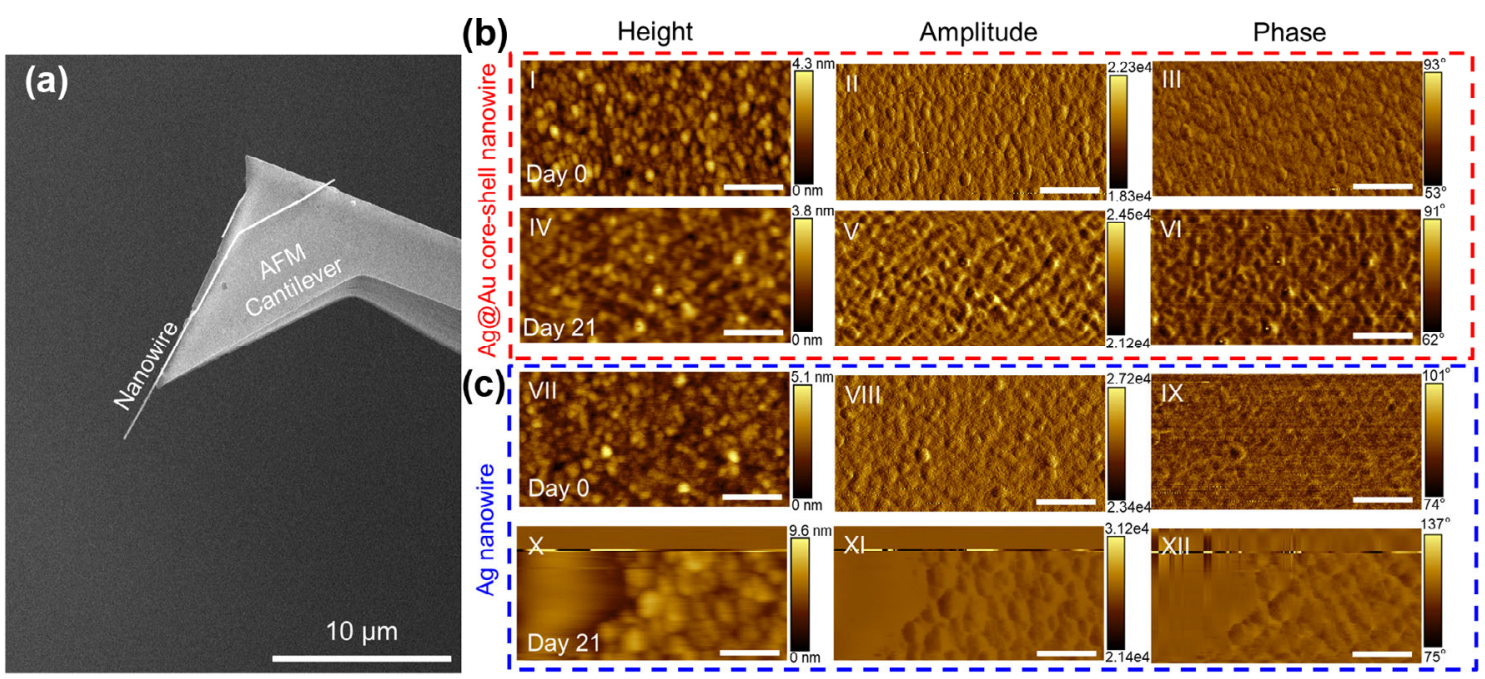

Figure 7 Tapping-mode AFM topography images of the 40-nm thick gold film E-beam evaporated on a quartz substrate. (a) SEM image of a nanowire-AFM probe. (b) AFM image scanned by the AgAuNW-AFM probe. Height, amplitude, phase imaging data were obtained by the AgAuNW-AFM probe that is freshly prepared (I, II, III) and after 21-days of exposure to air (IV, V, VI). (c) AFM image scanned by the pristine AgNW-AFM probe. Height, amplitude, phase imaging data obtained by the pristine AgNW-AFM probe that is freshly prepared (VII, VIII, IX) and after 21-days of exposure to air (X, XI, XII). All scale bars are $200 \mathrm{~nm}$.

performance after exposure to air for 21 days with a consistent $\sim 5 \mathrm{~nm}$ throughout the testing period (Fig. 7(b), bottom panel and Fig. S7 in the ESM), demonstrating the efficacy of the 3-nm epitaxial Au coating in prolonging the lifetime and enhancing the performance stability of AgNW SPM probes, even under high mechanical stress.

\section{Conclusions}

In summary, epitaxial Ag@Au core-shell nanowires with smooth surfaces and Au-Ag interfaces were synthesized with a simple room-temperature, solution-phase process that is highly compatible with large-scale, cost-effective device fabrication. The introduction of sulfite reduces the reduction potential of gold precursor and the potent reducing agent to suppress galvanic replacement and oxidative etching of the AgNW surface, resulting in a conformal, smooth, and even gold shell. By varying the reaction, the thickness of the ultrathin gold shell thickness can be precisely controlled to meet the requirement of various applications. The high-quality gold coating, even at only 3-nm shell thickness, proves highly effective in protecting the AgNW against oxidation under air, heat, humidity, light, mechanical stress, and in oxidative solutions. The Ag@Au core-shell nanowires demonstrate comparable performance with pristine AgNW in various electrical, optical, and mechanical devices with significant improvement in device stability and lifetime, offering a versatile and universal solution towards high-performance and high-durability AgNW devices for a wide variety of applications.

\section{Acknowledgements}

This material is based upon work supported by the National Science Foundation under gant No. CHE-1654794. The authors acknowledge Prof. Yadong Yin from the Department of Chemistry, UC Riverside for helpful discussion.

Electronic Supplementary Material: Supplementary material (SEM images of Ag@Au core-shell nanowires synthesized with different molar ratios between Au precursor and $\mathrm{Na}_{2} \mathrm{SO}_{3}$; EDS mapping image of Ag@Au core-shell nanowire ( $6 \mathrm{~nm} \mathrm{Au})$; SERS of 4-ATP functioned AgNWs and Ag@Au core-shell nanowires; AFM images scanned by AgNW (or Ag@Au core-shell nanowire)-AFM probe; TEM image of the end part of Ag@Au core-shell nanowire and reference) is available in the online version of this article at https://doi.org/10.1007/10.1007/s12274021-3718-z.

Open Access This article is licensed under a Creative Commons Attribution 4.0 International License, which permits use, sharing, adaptation, distribution and reproduction in any medium or format, as long as you give appropriate credit to the original author(s) and the source, provide a link to the Creative Commons licence, and indicate if changes were made.

The images or other third party material in this article are included in the article's Creative Commons licence, unless indicated otherwise in a credit line to the material. If material is not included in the article's Creative Commons licence and your intended use is not permitted by statutory regulation or exceeds the permitted use, you will need to obtain permission directly from the copyright holder.

To view a copy of this licence, visit http://creativecommons.org/licenses/by/4.0/.

\section{References}

[1] Li, W. W.; Yang, S.; Shamim, A. Screen printing of silver nanowires: Balancing conductivity with transparency while maintaining flexibility and stretchability. npj Flex. Electron. 2019, 3, 13.

[2] Lee, E.; Ahn, J.; Kwon, H. C.; Ma, S.; Kim, K.; Yun, S.; Moon, J. All-solution-processed silver nanowire window electrode-based flexible perovskite solar cells enabled with amorphous metal oxide protection. Adv. Energy Mater. 2018, 8, 1702182.

[3] Chen, C. R.; Qin, H. L.; Cong, H. P.; Yu, S. H. A highly stretchable and real-time healable supercapacitor. Adv. Mater. 2019, 31, 1900573.

[4] Ma, Z. L.; Kang, S. L.; Ma, J. Z.; Shao, L.; Wei, A. J.; Liang, C. B.; Gu, J. W.; Yang, B.; Dong, D. D.; Wei, L. F. et al. High-performance and rapid-response electrical heaters based on ultraflexible, heat-resistant, and mechanically strong aramid nanofiber/Ag nanowire nanocomposite papers. ACS Nano 2019, 13, 7578-7590.

[5] Chung, W. H.; Park, S. H.; Joo, S. J.; Kim, H. S. UV-assisted flash light welding process to fabricate silver nanowire/graphene on a PET substrate for transparent electrodes. Nano Res. 2018, 11, 2190-2203.

[6] Araki, T.; Jiu, J. T.; Nogi, M.; Koga, H.; Nagao, S.; Sugahara, T.; Suganuma, K. Low haze transparent electrodes and highly conducting 
air dried films with ultra-long silver nanowires synthesized by one-step polyol method. Nano Res. 2014, 7, 236-245.

[7] Kim, S.; Yan, R. X. Recent developments in photonic, plasmonic and hybrid nanowire waveguides. J. Mater. Chem. C 2018, 6, 1179511816.

[8] Qin, Q. Q.; Yin, S.; Cheng, G. M.; Li, X. Y.; Chang, T. H.; Richter, G.; Zhu, Y.; Gao, H. J. Recoverable plasticity in penta-twinned metallic nanowires governed by dislocation nucleation and retraction. Nat. Commun. 2015, 6, 5983.

[9] Jo, H. S.; An, S.; Park, C. W.; Woo, D. Y.; Yarin, A. L.; Yoon, S. S. Wearable, stretchable, transparent all-in-one soft sensor formed from supersonically sprayed silver nanowires. ACS Appl. Mater. Interfaces 2019, 11, 40232-40242.

[10] Kim, T.; Kang, S.; Heo, J.; Cho, S.; Kim, J. W.; Choe, A.; Walker, B.; Shanker, R.; Ko, H.; Kim, J. Y. Nanoparticle-enhanced silver-nanowire plasmonic electrodes for high-performance organic optoelectronic devices. Adv. Mater. 2018, 30, 1800659.

[11] Liu, Q. S.; Kim, S.; Ma, X. Z.; Yu, N.; Zhu, Y. Z.; Deng, S. Y.; Yan, R. X.; Zhao, H. J.; Liu, M. Ultra-sharp and surfactant-free silver nanowire for scanning tunneling microscopy and tip-enhanced Raman spectroscopy. Nanoscale 2019, 11, 7790-7797.

[12] Liu, J. W.; Wang, J. L.; Huang, W. R.; Yu, L.; Ren, X. F.; Wen, W. C.; $\mathrm{Yu}, \mathrm{S}$. H. Ordering Ag nanowire arrays by a glass capillary: A portable, reusable and durable SERS substrate. Sci. Rep. 2012, $2,987$.

[13] Kim, S.; Yu, N.; Ma, X. Z.; Zhu, Y. Z.; Liu, Q. S.; Liu, M.; Yan, R. $\mathrm{X}$. High external-efficiency nanofocusing for lens-free near-field optical nanoscopy. Nat. Photonics 2019, 13, 636-643.

[14] Ma, X. Z.; Zhu, Y. Z.; Yu, N.; Kim, S.; Liu, Q. S.; Apontti, L.; Xu, D.; Yan, R. X.; Liu, M. Toward high-contrast atomic force microscopytip-enhanced Raman spectroscopy imaging: Nanoantenna-mediated remote-excitation on sharp-tip silver nanowire probes. Nano Lett. 2019, 19, 100-107.

[15] Ma, X. Z.; Zhu, Y. Z.; Kim, S.; Liu, Q. S.; Byrley, P.; Wei, Y.; Zhang, J.; Jiang, K. L.; Fan, S. S.; Yan, R. X. et al. Sharp-tip silver nanowires mounted on cantilevers for high-aspect-ratio high-resolution imaging. Nano Lett. 2016, 16, 6896-6902.

[16] Choo, D. C.; Kim, T. W. Degradation mechanisms of silver nanowire electrodes under ultraviolet irradiation and heat treatment. Sci. Rep. 2017, 7, 1696.

[17] Elechiguerra, J. L.; Larios-Lopez, L.; Liu, C.; Garcia-Gutierrez, D.; Camacho-Bragado, A.; Yacaman, M. J. Corrosion at the nanoscale: The case of silver nanowires and nanoparticles. Chem. Mater. 2005, 17, 6042-6052.

[18] Joo, Y.; Byun, J.; Seong, N.; Ha, J.; Kim, H.; Kim, S.; Kim, T.; Im, H.; Kim, D.; Hong, Y. Silver nanowire-embedded PDMS with a multiscale structure for a highly sensitive and robust flexible pressure sensor. Nanoscale 2015, 7, 6208-6215.

[19] Li, Y. X.; Han, D. Y.; Jiang, C. J.; Xie, E. Q.; Han, W. H. A facile realization scheme for tactile sensing with a structured silver nanowire-PDMS composite. Adv. Mater. Technol. 2019, 4, 1800504.

[20] Oh, J. Y.; Lee, D.; Hong, S. H. Ice-templated bimodal-porous silver nanowire/PDMS nanocomposites for stretchable conductor. ACS Appl. Mater. Interfaces 2018, 10, 21666-21671.

[21] Dan, L.; Shi, S.; Chung, H. J.; Elias, A. Porous polydimethylsiloxanesilver nanowire devices for wearable pressure sensors. ACS Appl. Nano Mater. 2019, 2, 4869-4878.

[22] Cheng, C.; Xu, X. H.; Lei, H. X.; Li, B. J. Plasmon-assisted trapping of nanoparticles using a silver-nanowire-embedded PMMA nanofiber. Sci. Rep. 2016, 6, 20433.

[23] Li, W. W.; Meredov, A.; Shamim, A. Coat-and-print patterning of silver nanowires for flexible and transparent electronics. Npj Flex. Electron. 2019, 3, 19.

[24] Nair, N. M.; Pakkathillam, J. K.; Kumar, K.; Arunachalam, K.; Ray, D.; Swaminathan, P. Printable silver nanowire and PEDOT:PSS nanocomposite ink for flexible transparent conducting applications. ACS Appl. Electron. Mater. 2020, 2, 1000-1010.

[25] Thomas, J. P.; Rahman, M. A.; Srivastava, S.; Kang, J. S.; McGillivray, D.; Abd-Ellah, M.; Heinig, N. F.; Leung, K. T. Highly conducting hybrid silver-nanowire-embedded poly (3,4-ethylenedioxythiophene): Poly (styrenesulfonate) for high-efficiency planar silicon/organic heterojunction solar cells. ACS Nano 2018, 12, 9495-9503.

[26] Li, S. Y.; Chen, S. J.; Zhuo, B. G.; Li, Q. F.; Liu, W. J.; Guo, X. J.
Flexible ammonia sensor based on PEDOT:PSS/Silver nanowire composite film for meat freshness monitoring. IEEE Electron Device Lett. 2017, 38, 975-978.

[27] Ricciardulli, A. G.; Yang, S.; Wetzelaer, G. J. A. H.; Feng, X. L.; Blom, P. W. M. Hybrid silver nanowire and graphene-based solution-processed transparent electrode for organic optoelectronics. Adv. Funct. Mater. 2018, 28, 1706010.

[28] Yang, Y.; Chen, S.; Li, W. L.; Li, P.; Ma, J. G.; Li, B. S.; Zhao, X. N.; Ju, Z. S.; Chang, H. C.; Xiao, L. et al. Reduced graphene oxide conformally wrapped silver nanowire networks for flexible transparent heating and electromagnetic interference shielding. ACS Nano 2020, 14, 8754-8765.

[29] Cao, M. H.; Wang, M. Q.; Li, L.; Qiu, H. W.; Padhiar, M. A.; Yang, Z. Wearable rGO-Ag NW@cotton fiber piezoresistive sensor based on the fast charge transport channel provided by Ag nanowire. Nano Energy 2018, 50, 528-535.

[30] Huang, G. W.; Li, N.; Liu, Y.; Qu, C. B.; Feng, Q. P.; Xiao, H. M. Binder-free graphene/silver nanowire gel-like composite with tunable properties and multifunctional applications. ACS Appl. Mater. Interfaces 2019, 11, 15028-15037.

[31] Fang, Y. S.; Wu, Z. C.; Li, J.; Jiang, F. Y.; Zhang, K.; Zhang, Y. L.; Zhou, Y. H.; Zhou, J.; Hu, B. High-performance hazy silver nanowire transparent electrodes through diameter tailoring for semitransparent photovoltaics. Adv. Funct. Mater. 2018, 28, 1705409.

[32] Park, M.; Lee, S. H.; Kim, D.; Kang, J.; Lee, J. Y.; Han, S. M. Fabrication of a combustion-reacted high-performance $\mathrm{ZnO}$ electron transport layer with silver nanowire electrodes for organic solar cells. ACS Appl. Mater. Interfaces 2018, 10, 7214-7222.

[33] Kang, H.; Choi, S. R.; Kim, Y. H.; Kim, J. S.; Kim, S.; An, B. S.; Yang, C. W.; Myoung, J. M.; Lee, T. W.; Kim, J. G. et al. Electroplated silver-nickel core-shell nanowire network electrodes for highly efficient perovskite nanoparticle light-emitting diodes. ACS Appl. Mater. Interfaces 2020, 12, 39479-39486.

[34] Zhang, L. W.; Ji, Y.; Qiu, Y. J.; Xu, C. W.; Liu, Z. G.; Guo, Q. Q. Highly thermal-stable and transparent silver nanowire conductive films via magnetic assisted electrodeposition of Ni. J. Mater. Chem. C 2018, 6, 4887-4894.

[35] Zhou, K. L.; Zhang, Q. Q.; Wang, Z. L.; Wang, C. H.; Han, C. B.; Ke, X. X.; Zheng, Z. L.; Wang, H.; Liu, J. B.; Yan, H. A setariainflorescence-structured catalyst based on nickel-cobalt wrapped silver nanowire conductive networks for highly efficient hydrogen evolution. J. Mater. Chem. A 2019, 7, 26566-26573.

[36] Martinez, P. M.; Ishteev, A.; Fahimi, A.; Velten, J.; Jurewicz, I.; Dalton, A. B.; Collins, S.; Baughman, R. H.; Zakhidov, A. A. Silver nanowires on carbon nanotube aerogel sheets for flexible, transparent electrodes. ACS Appl. Mater. Interfaces 2019, 11, 32235-32243.

[37] Lee, J.; Woo, J. Y.; Kim, J. T.; Lee, B. Y.; Han, C. S. Synergistically enhanced stability of highly flexible silver nanowire/carbon nanotube hybrid transparent electrodes by plasmonic welding. ACS Appl. Mater. Interfaces 2014, 6, 10974-10980.

[38] Sun, Y. N.; Chang, M. J.; Meng, L. X.; Wan, X. J.; Gao, H. H.; Zhang, Y. M.; Zhao, K.; Sun, Z. H.; Li, C. X.; Liu, S. R. et al. Flexible organic photovoltaics based on water-processed silver nanowire electrodes. Nat. Electron. 2019, 2, 513-520.

[39] Khan, A.; Nguyen, V. H.; Muñoz-Rojas, D.; Aghazadehchors, S.; Jiménez, C.; Nguyen, N. D.; Bellet, D. Stability enhancement of silver nanowire networks with conformal $\mathrm{ZnO}$ coatings deposited by atmospheric pressure spatial atomic layer deposition. ACS Appl. Mater. Interfaces 2018, 10, 19208-19217.

[40] Nguyen, V. H.; Resende, J.; Papanastasiou, D. T.; Fontanals, N.; Jiménez, C.; Muñoz-Rojas, D.; Bellet, D. Low-cost fabrication of flexible transparent electrodes based on $\mathrm{Al}$ doped $\mathrm{ZnO}$ and silver nanowire nanocomposites: Impact of the network density. Nanoscale 2019, 11, 12097-12107.

[41] Zhao, Y.; Wang, X. J.; Yang, S. Z.; Kuttner, E.; Taylor, A. A.; Salemmilani, R.; Liu, X.; Moskovits, M.; Wu, B. H.; Dehestani, A. et al. Protecting the nanoscale properties of ag nanowires with a solution-grown $\mathrm{SnO}_{2}$ monolayer as corrosion inhibitor. J. Am. Chem. Soc. 2019, 141, 13977-13986.

[42] Yang, Y.; Dong, R. Z.; Zhu, Y. L.; Li, H. S.; Zhang, H.; Fan, X. M.; Chang, H. L. High-performance direct hydrogen peroxide fuel cells (DHPFCs) with silver nanowire-graphene hybrid aerogel as highly- 
conductive mesoporous electrodes. Chem. Eng. J. 2020, 381, 122749.

[43] Yang, M. X.; Hood, Z. D.; Yang, X.; Chi, M. F.; Xia, Y. N. Facile synthesis of $\mathrm{Ag} @ \mathrm{Au}$ core-sheath nanowires with greatly improved stability against oxidation. Chem. Commun. 2017, 53, 1965-1968.

[44] Choi, S.; Han, S. I.; Jung, D.; Hwang, H. J.; Lim, C.; Bae, S.; Park, O. K.; Tschabrunn, C. M.; Lee, M.; Bae, S. Y. et al. Highly conductive, stretchable and biocompatible $\mathrm{Ag}-\mathrm{Au}$ core-sheath nanowire composite for wearable and implantable bioelectronics. Nat. Nanotechnol. 2018, $13,1048-1056$.

[45] Huang, Z. L.; Meng, G. W.; Hu, X. Y.; Pan, Q. J.; Huo, D. X.; Zhou, H. J.; Ke, Y.; Wu, N. Q. Plasmon-tunable Au@Ag core-shell spiky nanoparticles for surface-enhanced Raman scattering. Nano Res. 2019, 12, 449-455.

[46] Khlebtsov, B.; Khanadeev, V.; Khlebtsov, N. Surface-enhanced Raman scattering inside $\mathrm{Au} @ \mathrm{Ag}$ core/shell nanorods. Nano Res. 2016, 9, 2303-2318.

[47] Wang, D. M.; Hua, H. M.; Liu, Y.; Tang, H. R.; Li, Y. X. Single Ag nanowire electrodes and single Pt@Ag nanowire electrodes: Fabrication, electrocatalysis, and surface-enhanced Raman scattering applications. Anal. Chem. 2019, 91, 4291-4295.

[48] Zhang, L.; Zhang, Y.; Ahn, J.; Wang, X.; Qin, D. Defect-assisted deposition of $\mathrm{Au}$ on $\mathrm{Ag}$ for the fabrication of core-shell nanocubes with outstanding chemical and thermal stability. Chem. Mater. 2019 , 31, 1057-1065.

[49] Au, L.; Lu, X. M.; Xia, Y. N. A comparative study of galvanic replacement reactions involving $\mathrm{Ag}$ nanocubes and $\mathrm{AuCl}_{2}{ }^{-}$or $\mathrm{AuCl}_{4}^{-}$. Adv. Mater. 2008, 20, 2517-2522.

[50] Niu, Z. Q.; Cui, F.; Yu, Y.; Becknell, N.; Sun, Y. C.; Khanarian, G.; Kim, D.; Dou, L.; Dehestani, A.; Schierle-Arndt, K. et al. Ultrathin epitaxial Cu@Au core-shell nanowires for stable transparent conductors. J. Am. Chem. Soc. 2017, 139, 7348-7354.

[51] Gao, C. B.; Lu, Z. D.; Liu, Y.; Zhang, Q.; Chi, M. F.; Cheng, Q.; Yin, Y. D. Highly stable silver nanoplates for surface plasmon resonance biosensing. Angew. Chem., Int. Ed. 2012, 51, 5629-5633.

[52] Xue, C.; Chen, X.; Hurst, S. J.; Mirkin, C. A. Self-assembled monolayer mediated silica coating of silver triangular nanoprisms. Adv. Mater. 2007, 19, 4071-4074

[53] Gao, C. B.; Zhang, Q.; Lu, Z. D.; Yin, Y. D. Templated synthesis of metal nanorods in silica nanotubes. J. Am. Chem. Soc. 2011, 133, 19706-19709.

[54] Hu, L. B.; Kim, H. S.; Lee, J. Y.; Peumans, P.; Cui, Y. Scalable coating and properties of transparent, flexible, silver nanowire electrodes. ACS Nano 2010, 4, 2955-2963.

[55] Liu, H. P.; Liu, T. Z.; Zhang, L.; Han, L.; Gao, C. B.; Yin, Y. D. Etching-free epitaxial growth of gold on silver nanostructures for high chemical stability and plasmonic activity. Adv. Funct. Mater. 2015, 25, 5435-5443.

[56] Yang, Y.; Liu, J. Y.; Fu, Z. W.; Qin, D. Galvanic replacement-free deposition of $\mathrm{Au}$ on $\mathrm{Ag}$ for core-shell nanocubes with enhanced chemical stability and SERS activity. J. Am. Chem. Soc. 2014, 136, 8153-8156.

[57] Choi, Y.; Hong, S.; Liu, L. C.; Kim, S. K.; Park, S. Galvanically replaced hollow $\mathrm{Au}-\mathrm{Ag}$ nanospheres: Study of their surface plasmon resonance. Langmuir 2012, 28, 6670-6676.

[58] Cui, F.; Yu, Y.; Dou, L. T.; Sun, J. W.; Yang, Q.; Schildknecht, C.; Schierle-Arndt, K.; Yang, P. D. Synthesis of ultrathin copper nanowires using tris(trimethylsilyl)silane for high-performance and low-haze transparent conductors. Nano Lett. 2015, 15, 7610-7615.

[59] Rathmell, A. R.; Wiley, B. J. The synthesis and coating of long, thin copper nanowires to make flexible, transparent conducting films on plastic substrates. Adv. Mater. 2011, 23, 4798-4803.

[60] Hecht, D. S.; Heintz, A. M.; Lee, R.; Hu, L. B.; Moore, B.; Cucksey, C.; Risser, S. High conductivity transparent carbon nanotube films deposited from superacid. Nanotechnology 2011, 22, 075201.

[61] Vosgueritchian, M.; Lipomi, D. J.; Bao, Z. N. Highly conductive and transparent PEDOT:PSS films with a fluorosurfactant for stretchable and flexible transparent electrodes. Adv. Funct. Mater. 2012, 22, 421-428.

[62] Ye, S. R.; Rathmell, A. R.; Stewart, I. E.; Ha, Y. C.; Wilson, A. R.; Chen, Z. F.; Wiley, B. J. A rapid synthesis of high aspect ratio copper nanowires for high-performance transparent conducting films. Chem. Commun. 2014, 50, 2562-2564.

[63] Yan, R. X.; Pausauskie, P.; Huang, J. X.; Yang, P. D. Direct photonic-plasmonic coupling and routing in single nanowires. Proc. Natl. Acad. Sci. USA 2009, 106, 21045-21050.

[64] Kim, S.; Bailey, S.; Liu, M.; Yan, R. X. Decoupling co-existing surface plasmon polariton (SPP) modes in a nanowire plasmonic waveguide for quantitative mode analysis. Nano Res. 2017, 10, 2395-2404.

[65] Yan, R. X.; Park, J. H.; Choi, Y.; Heo, C. J.; Yang, S. M.; Lee, L. P.; Yang, P. D. Nanowire-based single-cell endoscopy. Nat. Nanotechnol. 2012, 7,191-196.

[66] Lee, W.; Kang, B. H.; Yang, H.; Park, M.; Kwak, J. H.; Chung, T.; Jeong, Y.; Kim, B. K.; Jeong, K. H. Spread spectrum SERS allows label-free detection of attomolar neurotransmitters. Nat. Commun. 2021, 12, 159.

[67] Yeh, Y. T.; Gulino, K.; Zhang, Y. H.; Sabestien, A.; Chou, T. W.; Zhou, B.; Lin, Z.; Albert, I.; Lu, H. G.; Swaminathan, V. et al. A rapid and label-free platform for virus capture and identification from clinical samples. Proc. Natl. Acad. Sci. USA 2020, 117, 895-901. 